Perspective

\title{
Emergence of novel coronavirus: global context
}

Keiji Fukuda ${ }^{1}$

In the past four decades, the emergence of new infectious diseases has shaped not only medical concepts, but also those of science and public health; affected political responses at global, regional and national levels; had serious economic impact; and influenced the anxieties and expectations of the public. The novel coronavirus ( $\mathrm{nCoV}$ ) discovered in 2012 adds another such disease [1] and its potential impact may best be understood in such a historic and global context.

In the 1980s, the emergence of HIV/AIDS had an enormous impact on medicine, science, politics and society-an impact that is still unfolding. The concerns raised by HIV/AIDS changed the relationship of patients to their disease, treatment and physicians and provided the clearest example to date that a disease has the capacity to become a globally transforming political issue, affecting national and international relations [2].

The decade from 2000 to 2010 was an active one for emerging infectious diseases. The emergence of severe acute respiratory syndrome (SARS) demonstrated the speed at which an infectious disease, in this globalized world, can move beyond its local origins to become a global crisis affecting the health of people and economies by reducing international travel and trade. The emergence of other infectious-disease agents, such as avian influenza $\mathrm{A}(\mathrm{H} 5 \mathrm{Nl})$ and Nipah virus, further emphasized the inextricable links between human health, agriculture and the environment.

SARS and H5N1 became the driving forces for countries to reshape the International Health Regulations (IHR) and to adopt a revision in 2005. The regulations, which came into force in 2007, are an acknowledgement that all countries are at risk from certain threats, such as a new infectious disease with the potential for international spread [3]. The IHR also stress the need for a proactive approach by affected countries and the need for transparency in reporting. This approach encompasses prevention, containment, investigation and timely reporting of findings.

In 2011, a new fundamental public health agreement called the Pandemic Influenza Preparedness (PIP) Framework was adopted after more than four years of formal negotiations. This Framework was catalysed by the spread of $\mathrm{H} 5 \mathrm{N1}$, which sharply demonstrated the need for equity among countries [4]. In a strict sense, the PIP Framework pertains only to potential pandemic influenza viruses but reflects the larger concerns of Member States of the World Health Organization (WHO).

Under the PIP Framework, countries have agreed that the sharing of potential pandemic influenza viruses as well as the benefits resulting from this sharing, such as diagnostic tests, vaccines and medicines, are of equal importance. The discussions preceding adoption of the Framework highlighted other problematic issues around activities, such as research, publications, material transfer agreements, and patents and other intellectual property claims. These issues can become potential impediments to the sharing of critical information, materials and technology. Moreover, it is clear that the full implementation of this Framework, similar to the IHR, will take time. Nonetheless, both frameworks clearly point to the directions that the world will need to move if such new diseases and other risks to global health security are to be optimally addressed.

The 2009 H1N1 influenza pandemic demonstrated that a global outbreak of even a relatively mild disease could overwhelm the capacity of many countries to respond and raised a number of issues. At the beginning of the pandemic, the naming of the virus raised concerns with respect to fairness and stigmatization. The naming issue, along with the rapid pace of developments and enormous amounts of information and misinformation, aided in particular by social media and the Internet, created significant levels of distrust and anxiety among countries, the media, the public, individuals and organizations. The pandemic eventually underscored that countries are better prepared than in the past but they still have much to do to be adequately prepared.

With the emergence of a novel coronavirus in 2012, we are once again in a situation both familiar and uncertain. This novel virus is capable of causing severe disease and death. Evidence suggests that limited human-to-human transmission has occurred but its prospects for sustained or easy transmission remain unresolved. In addition, the source - presumably an animal - is still unknown, complicating efforts to limit exposure. In short, the future course is uncertain but it is clear that early decisions and actions could have profound and global effects. 
When new threats to human health arise, anxiety, needs and expectations are high. Policy-makers require information quickly that will inform risk assessments and potential countermeasures. The public, likewise, demand transparency and trust and assurance that the actions taken by all of those involved, including WHO and its Member States, scientific and public health organizations, and the research community, are guided by overarching concerns about global health and security and commitment to the highest ideals of science, fairness and equity. In short, the principles embodied in both the IHR and the PIP Framework.

Just as events such as the emergence of a novel pathogen have international consequences, successfully responding to them requires international cooperation. This includes information sharing and cooperation between animal and human health sectors, between ministries of health in affected countries and between those conducting research and those on the front lines. Bringing diverse expertise and moving information quickly to those who need to know to address the critical questions related to the novel pathogen is critical for informing control efforts. The IHR provide both a useful mechanism for reporting and sharing information through WHO, and a framework for making decisions about what to report. As has been seen in SARS, H5N1, Nipah and countless other emerging pathogens, WHO serves a critical coordinating function, providing a platform for this coordination and collaboration, and synthesizing information into useful guidance for Member States.

\section{References}

1. Zaki AM et al. Isolation of a novel coronavirus from a man with pneumonia in Saudi Arabia. New England Journal of Medicine, 2012, 367:1814-1820.

2. De Cock KM et al. Reflections on 30 years of AIDS. Emerging Infectious Diseases, 2011, 17(6):1044-1048.
3. International Health Regulations, 2005, 2nd ed. Geneva, World Health Organization, 2008.

4. Pandemic influenza preparedness framework for the sharing of influenza viruses and access to vaccines and other benefits. Geneva: World Health Organization, 2011. 